\title{
A rare case of amelanotic malignant melanoma in the oral region: Clinical investigation and immunohistochemical study
}

\author{
YUICHI OHNISHI ${ }^{1}$, MASAHIRO WATANABE ${ }^{1}$, TOMOKO FUJII ${ }^{1}$, NORIKO SUNADA ${ }^{1}$, \\ HITOSHI YOSHIMOTO $^{1}$, HIROHITO KUBO ${ }^{1}$, MASAHIRO WATO ${ }^{2}$ and KENJI KAKUDO ${ }^{1}$ \\ ${ }^{1}$ Second Department of Oral and Maxillofacial Surgery, Osaka Dental University, Chuo-ku, Osaka 540-0008; \\ ${ }^{2}$ Department of Oral Pathology, Osaka Dental University, Hirakata-shi, Osaka 573-1121, Japan
}

Received September 16, 2014; Accepted June 22, 2015

DOI: $10.3892 / 01.2015 .3819$

\begin{abstract}
Amelanotic malignant melanoma (AMM) is rare in the oral region. The present study examined the clinical features of this tumor in an attempt to establish diagnostic criteria. The expression of three melanocytic differentiation markers, HMB-45, S-100 and Melan-A, was also measured in primary oral AMMs in order to determine whether the markers could be used to diagnose primary oral AMMs and to find out which marker was the most sensitive. It may be particularly difficult to correctly diagnose AMM that lacks a radial growth phase without immunohistochemical assistance. In the present study, mixtures of polygonal and spindle cells at different ratios were observed in the tumors with and without a radial growth phase. Immunohistochemistry was used to examine the HMB-45, S-100 and Melan-A expression in the formalin-fixed paraffin-embedded specimens of primary oral AMMs. Comparison of staining intensities (SIs) and labeling indices (LIs) of the markers was also performed. The immunostaining results revealed that the SI of Melan-A was significantly higher than that of $\mathrm{S}-100(\mathrm{P}=0.0011)$. HMB-45, S-100 and Melan-A also exhibited high positive rates and LIs in AMMs and, therefore, may be good markers for the immunohistochemical diagnosis of primary oral AMMs. Furthermore, Melan-A may be a more sensitive marker than S-100 and HMB-45, as it has a higher SI.
\end{abstract}

\section{Introduction}

Oral malignant melanomas (MMs) are extremely rare, accounting for $<2 \%$ of all reported MMs. MMs are known to have a high potential for malignancy, invasiveness and metastasis $(1,2)$. Although MMs with characteristic

Correspondence to: Dr Yuichi Ohnishi, Second Department of Oral and Maxillofacial Surgery, Osaka Dental University, 1-5-17 Otemae, Chuo-ku, Osaka 540-0008, Japan

E-mail: ohnishi@cc.osaka-dent.ac.jp

Key words: amelanotic malignant melanoma, oral, immunohistochemistry, HMB-45, S-100, Melan-A melanin-pigmented tumor cells are easy to diagnose, amelanotic MMs (AMMs) are extremely difficult to diagnosis due to the absence of these cells $(3,4)$. AMM can be divided into the following five types based on the varied clinical features observed: The pigmented nodular type, the non-pigmented nodular type, the pigmented macular type, the pigmented mixed type and the non-pigmented mixed type $(5,6)$. The non-pigmented nodular type is an amelanotic tumor that lacks a radial growth phase, while the non-pigmented mixed type is an amelanotic nodular tumor that is surrounded by a radial growth phase. It is hard to distinguish between amelanotic epithelioid melanoma and poorly-differentiated carcinoma or large cell lymphoma (4,7). Furthermore, the differentiation between spindle cell melanomas and sarcomas and sarcomatoid carcinomas is also difficult $(7,8)$. In such cases, immunohistochemistry with antibodies directed against certain melanocytic differentiation antigens is typically required for the final diagnosis $(4,7,8)$.

Currently, the three most useful immunomarkers for the identification of melanocytes and melanomas are HMB-45, S-100 and Melan-A (4,7-11). The S-100 protein is an acidic, calcium-binding protein that Moore (12) first extracted from the bovine brain in 1965. Although it is an extremely sensitive marker for nevi and melanoma cells (9), the central and peripheral nervous systems of all vertebrates also contain a wide distribution (13). A number of previous studies have used the anti-S-100 antibody to immunohistochemically diagnose the primary and metastatic MMs of various sites in the human body (14).

Anti-HMB-45 was initially described by Gown et al (15) in 1986. The antibody recognizes gp100, a melanosomal glycoprotein. The HMB-45 antigen has previously been shown to be localized in pre-melanosomes, with HMB-45-positive staining associated with active early melanosome formation (9).

In 1985, Melan-A was cloned by Coulie et al (16) from the human melanoma SK-MEL-29 cell line. Melan-A encodes a melanoma antigen that autologous cytotoxic $\mathrm{T}$ cells recognize. The Melan-A-derived peptide recognized by the $\mathrm{T}$ cells has been identified as the nonamer AAGIGILTV, which shows HLA-A2 restriction. Isolation of T cells with specificity for Melan-A is possible in normal individuals, as well as melanoma patients $(17,18)$. A previous study revealed that Melan-A-positive staining was associated with initially developed and massively proliferated lesions. In the present study, the expression of 
three melanocytic differentiation markers, HMB-45, S-100 and Melan-A, was examined by immunohistochemistry in order to determine whether HMB-45, S-100 and Melan-A were useful for diagnosing primary oral AMM. The study also attempted to identify which marker was the most sensitive. Written informed consent was obtained from the patient's family.

\section{Case report}

Patient. In October 2009, an 80-year-old male presented to the Second Department of Oral and Maxillofacial Surgery, Osaka Dental University (Chuo-ku, Japan) with a painful swelling in the left maxillary region that had been apparent for 1 month. The patient had undergone surgery for esophageal cancer three months earlier at a medical hospital. A physical examination revealed a $4 \times 5-\mathrm{cm}$ enlargement in the left maxillary gingiva that extended from the midline to the first molar. The lesion was mildly erythematous with ill-defined borders and an intact, smooth mucosal surface (Fig. 1). A radiographic investigation using orthopantomography revealed slightly horizontal bone resorption in the alveolar ridge. There was no palpable lymphadenopathy. An incisional biopsy was performed under local anesthesia. Microscopically, the cellularity was high, with an increased density of epithelioid-type and spindle-type cells. Moreover, there were also cells with bizarre nuclei, and notable mitosis (Fig. 2). Immunoreactivity was detected in the tumor cells for S-100, HMB-45 and Melan-A (Figs. 3-5). Therefore, the pathological diagnosis of the lesion was AMM. In December 2009, the patient underwent surgery (partial maxillectomy and bilateral modified radical neck dissection) and in January 2010 received systemic dacarbazine, nimustine and vincristine, and local intracutaneous interferon $\beta$ therapy (DAV-Feron) (19) at the Department of Otolaryngology and Head and Neck Surgery, Osaka City University Graduate School of Medicine (Osaka, Japan). After DAV-Feron, in August 2010, dentures were created at the Department of Geriatric Dentistry, Osaka Dental University.

Clinical investigation. Non-pigmented tumors without a radial growth phase have clinically been classified as the non-pigmented nodular type $(4,20)$. This type of tumor has often been misdiagnosed as an epulis, squamous cell carcinoma or benign tumor due to the lack of pigments and a radial growth phase. In the present case, the non-pigmented nodular type tumor was formed from spindle cells and a majority of polygonal cells. Only a small number of pigmented neoplastic melanocytes were observed. Hematoxylin and eosin staining indicated a histopathological diagnosis of MM. Immunohistochemical staining with antibodies against HMB-45, S-100, and Melan-A was positive, thus confirming the diagnosis.

Immunohistochemistry. Tissue samples were fixed in $10 \%$ neutral buffered formalin solution immediately after resection and then embedded in paraffin. Sections ( $4-\mu$ m thick) were cut and mounted on silane-coated glass slides. The sections were deparaffinized in L-limonene and dehydrated through a graded ethanol series. Antigen retrieval was performed by autoclaving at $121^{\circ} \mathrm{C}$ for $15 \mathrm{~min}$ in Histo VT One ${ }^{\circledast}(\mathrm{pH} 7.0$; Nacalai Tesque, Kyoto, Japan). Endogenous peroxidase activity was blocked with $3 \% \mathrm{H}_{2} \mathrm{O}_{2}$ for $10 \mathrm{~min}$. Tissue sections were then incubated

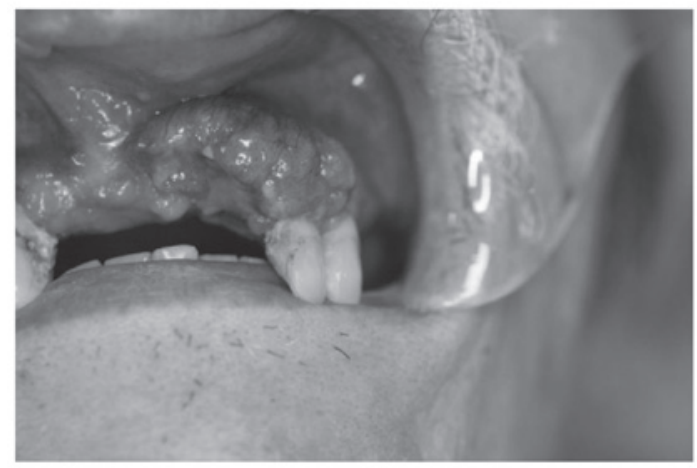

Figure 1. Intraoral view showing a non-pigmented lesion in the first visit.
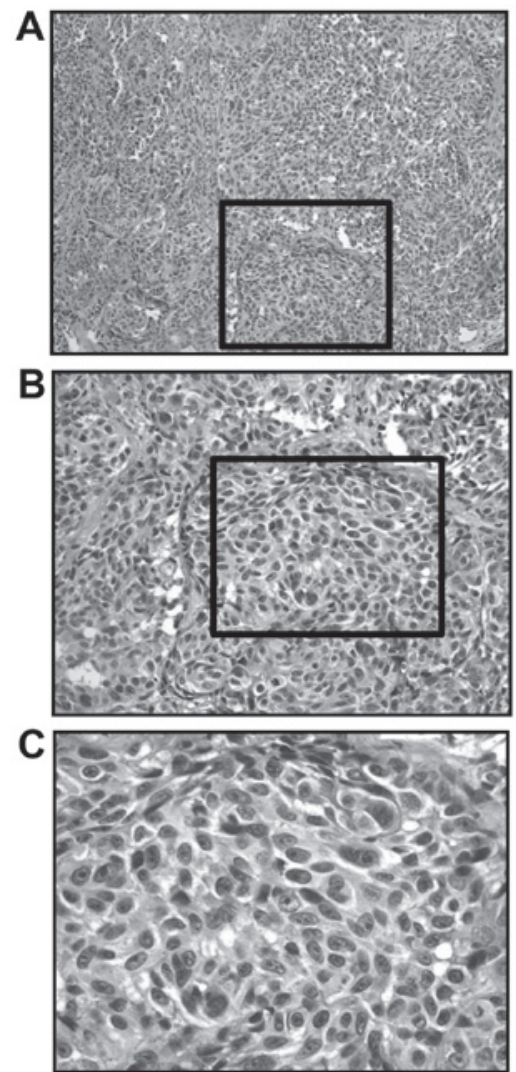

Figure 2. Microscopic findings of the tumor. Cellularity was high and the density of epithelioid-type and spindle-type cells was increased. Moreover, there were also cells with bizarre nuclei, and mitosis was marked. Original magnification, (A) x10, (B) x20 and (C) x40.

with the anti-HMB-45 monoclonal antibody (Dako, Tokyo, Japan), anti-S-100 polyclonal antibody (Nichirei Bioscience, Tokyo, Japan) and anti-Melan-A monoclonal antibody (Dako) at room temperature for $1 \mathrm{~h}$. The tissue slides were incubated with peroxidase micropolymer-conjugated secondary antibodies (Vector Laboratories, Burlingame, CA, USA) at room temperature for $30 \mathrm{~min}$, and visualized by incubation with the 3,3'-diaminobenzidine tetrahydrochloride liquid system (Dako) at room temperature for $5 \mathrm{~min}$. The sections were subsequently counterstained with hematoxylin and observed by light microscopy (Olympus Corporation, Tokyo, Japan). Cutaneous melanoma and nevus tissues, obtained during surgeries performed between April and October 2011 at Gokeikai Osaka Kaisei Hospital 


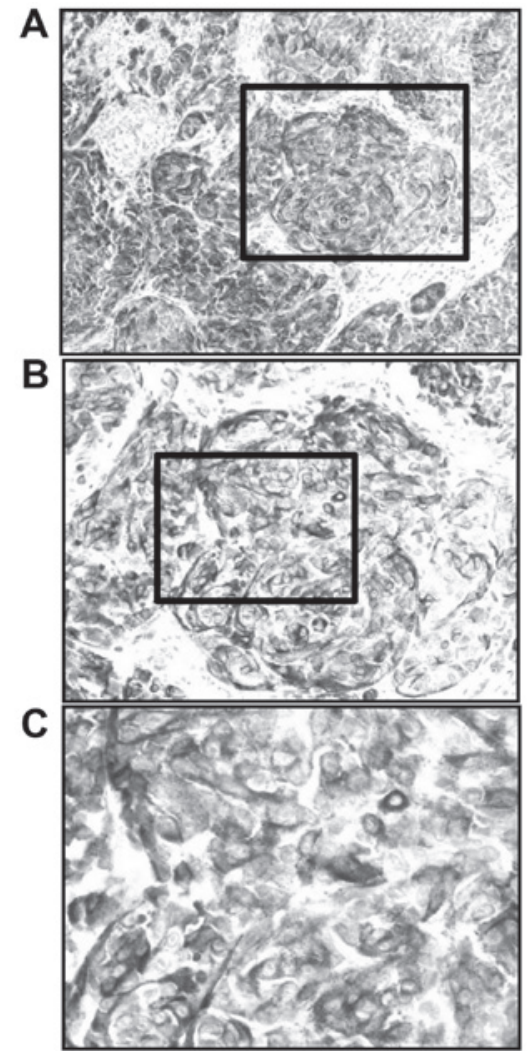

Figure 3. Tumor cells showing positive immunohistochemical staining for HMB-45. HMB-45 demonstrating strong brown cytoplasmic staining in all melanoma cells. Original magnification, (A) x10, (B) x20 and (C) x40.
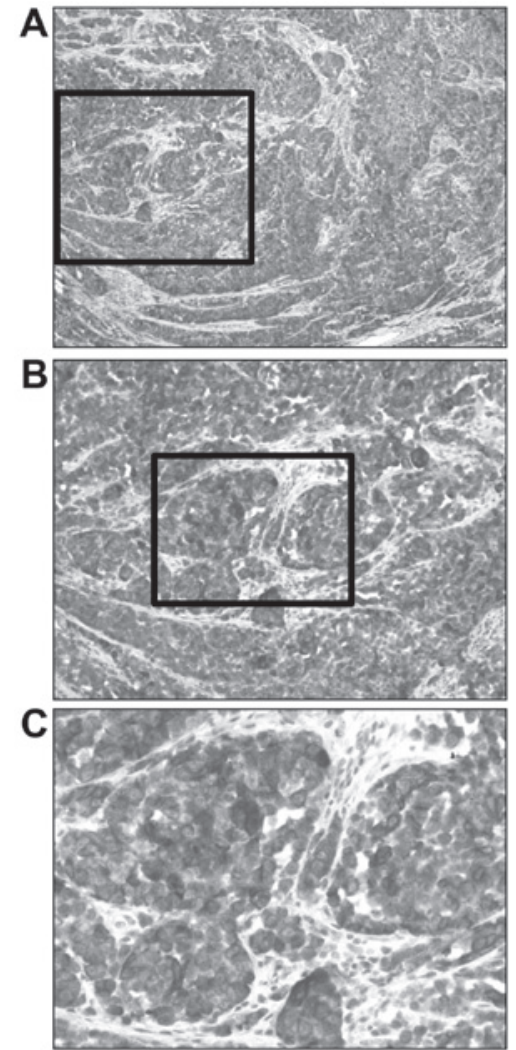

Figure 5. Tumor cells showing positive immunohistochemical staining for Melan-A. Melan-A showing strong brown cytoplasmic staining in nearly all melanoma cells. Original magnification, (A) x10, (B) x20 and (C) x40.

(Osaka, Japan), were provided by Dr. Masaki Akane (Gokeikai Osaka Kaisei Hospital) and served as positive controls, and Tris-buffered saline was used instead of primary antibody as the negative control. Brown cytoplasmic staining was considered as a positive result for HMB-45 and Melan-A, and brown nuclear and cytoplasmic staining was considered to be positive for the S-100 protein. Positively immunostained cells were easy to distinguish from melanin-pigmented cells when the sections were carefully compared with negative control sections. Staining intensities (SIs) were graded as follows: 0, no staining; 1, weak staining; 2 , moderate staining; and 3, strong staining, when compared with the cutaneous melanoma positive control sections, in which the SI was graded as 3. Labeling indices (LIs) were calculated by initially scanning the sections at low power, then selecting at least three high-power fields and counting 1,000 tumor cells in each of the three fields. HMB-45, S-100, and Melan-A LIs were counted as the ratio of positively immunostained tumor cells to the total number of tumor cells counted.

Statistical analysis. A Mann-Whitney U test was performed using the SPSS software package (version 13.0; SPSS, Inc., Chicago, IL, USA) to assess statistically significant differences between samples. Data are presented as the mean \pm standard deviation. $\mathrm{P}<0.05$ was considered to indicate a statistically significant difference.

Results. The majority of HMB-45, S-100 and Melan-A immunostaining in the melanoma cells was cytoplasmic, but nuclear staining was also found in the anti-S-100-stained
Figure 4. Tumor cells showing positive immunohistochemical staining for S-100. S-100 exhibiting moderate to strong brown cytoplasmic and nuclear staining in all melanoma cells. Original magnification (A) x10, (B) x20 and (C) $\mathrm{x} 40$.

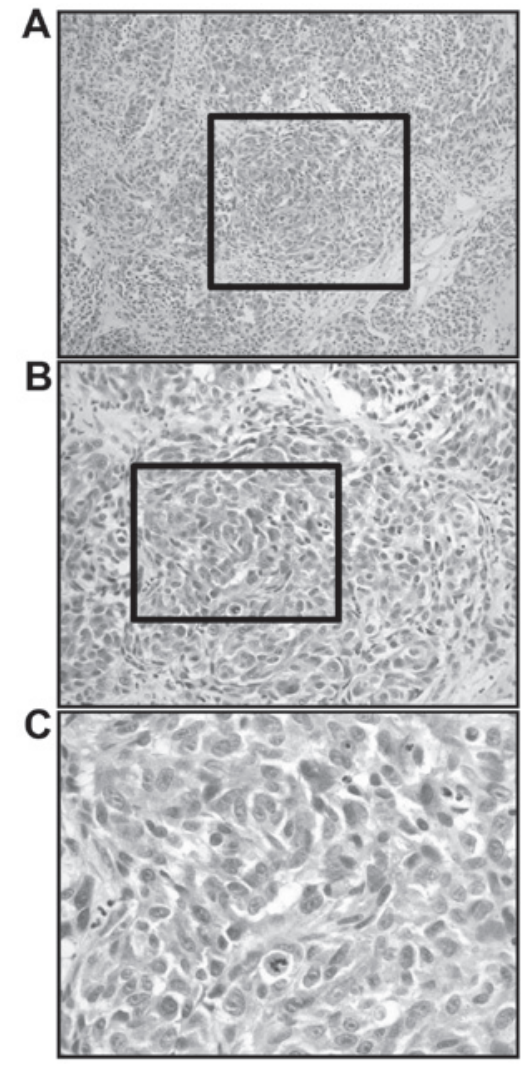


tumor cells. The SIs for HMB-45-positive AMM were graded as 3 (Fig. 3). The LI for HMB-45-positive AMM was 85\%. The S-100 SI was graded as 2 (Fig. 4) and the LI for S-100-positive AMM was $71 \%$. Additionally, the SIs for Melan-A-positive AMM were graded as 3 (Fig. 5) and the LI was $95 \%$.

\section{Discussion}

Oral MM accounts for only 0.2 to $8.0 \%$ of all MMs (1). The 5 -year survival rate has previously been reported to be between 15 and $38 \%$ (21). The origins of AMM reside in the malignant transformation of proliferating melanocytes existing in the epithelial basal layer in the mucosa. The palate is the most commonly affected site, followed by the maxillary gingiva (18). The differential diagnosis for pigmented lesions on the oral mucosa includes melanotic macule, nevi, melanoma, physiological pigmentation and tattoos (21). Moreover, as AMM accounts for only $2.3 \%$ of all MMs (22), it is extremely rare.

Clinically, the diagnosis of MM is commonly dependent on the presence of a pigmented lesion (23). In its absence, there is typically no other parameter to alert a clinician to the possibility of MM, particularly in the oral region due to its rarity in this location. Upon histopathological examination, a mixture of polygonal and spindle cells are present in differing proportions. Amelanotic or only slightly pigmented tumors can, however, be diagnosed correctly with immunohistochemistry. In the present study, immunohistochemical staining with antibodies against the HMB-45, S-100 and Melan-A proteins successfully diagnosed this amelanotic case. Anti-S-100, anti-HMB-45 and anti-Melan-A are the three most commonly used antibodies for the immunohistochemical diagnosis of primary oral MM. Previous studies have recorded a positive rate of $100 \%$ for anti-S-100 $(3,7,11)$ and $\sim 79 \%$ (range, $71-100 \%$ ) for anti-HMB-45 $(1,3,7,11$ ). These results indicated that $\mathrm{S}-100$ is a more sensitive marker than HMB-45 and Melan-A for immunohistochemically diagnosing MM. However, Naoki et al (24) reported that Melan-A-positive staining was associated with both initially developed and massively proliferated lesions. However, HMB-45 and S-100 were positive in initially developed lesions and negative in massively proliferated lesions. Furthermore, the SI of Melan-A was higher than those of HMB-45 and S-100 in the present study. Taken together, these results suggest that HMB-45, S-100, and Melan-A may be good markers for immunohistochemically diagnosing primary oral MMs. Moreover, Melan-A may be a more sensitive and specific marker than S-100 and HMB-45.

In conclusion, oral MMs are extremely rare and difficult to diagnosis due to the absence of melanin-pigmented tumor cells. The three most useful immunomarkers for the identification of melanocytes and melanomas appear to be HMB-45, S-100 and Melan-A. Furthermore, Melan-A may be a more sensitive and specific marker than S-100 and HMB-45.

\section{References}

1. Manganaro AM, Hammond HL, Dalton MJ and Williams TP: Oral melanoma: Case reports and review of the literature. Oral Surg Oral Med Oral Pathol Oral Radiol Endod 80: 670-676, 1995.

2. Rapini RP: Oral melanoma: Diagnosis and treatment. Semin Cutan Med Surg 16: 320-322, 1997.
3. Gazit D and Daniels TE: Oral melanocytic lesions: Differences in expression of HMB-45 and S-100 antigens in round and spindle cells of malignant and benign lesions. J Oral Pathol Med 23: 60-64, 1994.

4. Hirano T: Immunohistochemical study of malignant melanoma. Acta Pathol Jpn 36: 733-743, 1986.

5. Tanaka N, Amagasa T, Iwaki H, Shioda S, Takeda M, Ohashi K and Reck SF: Oral malignant melanoma in Japan. Oral Surg Oral Med Oral Pathol 78: 81-90, 1994.

6. Tanaka N, Mimura M, Ichinose S and Odajima T: Malignant melanoma in the oral region: Ultrastructural and immunohistochemical studies. Med Electron Microsc 34: 198-205, 2001.

7. Prasad ML, Jungbluth AA, Iversen K, Huvos AG and Busam KJ: Expression of melanocytic differentiation markers in malignant melanomas of the oral and sinonasal mucosa. Am J Surg Pathol 25: 782-787, 2001.

8. Kilpatrick SE, White WL and Browne JD: Desmoplastic malignant melanoma of the oral mucosa. An underrecognized diagnostic pitfall. Cancer 78: 383-389, 1996.

9. Blessing K, Sanders DS and Grant JJ: Comparison of immunohistochemical staining of the novel antibody melan-A with S100 protein and $\mathrm{HMB}-45$ in malignant melanoma and melanoma variants. Histopathology 32: 139-146, 1998.

10. Jungbluth AA, Busam KJ, Gerald WL, Stockert E, Coplan KA, Iversen K, MacGregor DP, Old LJ and Chen YT: A103: An anti-Melan-a monoclonal antibody for the detection of malignant melanoma in paraffin-embedded tissues. Am J Surg Pathol 22: 595-602, 1998.

11. Barrett AW, Bennett JH and Speight PM: A clinicopathological and immunohistochemical analysis of primary oral mucosal melanoma. Eur J Cancer B Oral Oncol 31B: 100-105, 1995.

12. Moore BW: A soluble protein characteristic of the nervous system. Biochem Biophys Res Commun 19: 739-744, 1965.

13. Nakajima T, Watanabe S, Sato Y, Kameya T, Shimosato Y and Ishihara K: Immunohistochemical demonstration of S100 protein in malignant melanoma and pigmented nevus and its diagnostic application. Cancer 50: 912-918, 1982.

14. Nobuyuki T, Teruo A, Hiroshi I, Shigetoshi S, Masamune T, Kenichi O and Steven F: Oral malignant melanoma in Japan. Oral Surg Oral Med Oral Pathol 78: 81-90, 1994.

15. Gown AM, Vogel AM, Hoak D, Gough F and McNutt MA: Monoclonal antibodies specific for melanocytic tumors distinguish subpopulations of melanocytes. Am J Pathol 123: 195-203, 1986.

16. Rapini RP, Golitz LE, Greer RO Jr, Krekorian EA and Poulson T: Primary malignant melanoma of the oral cavity: A review of 177 cases. Cancer 55: 1543-1551, 1985.

17. Sensi M, Traversari C, Radrizzani M, Salvi S, Maccalli C, Mortarini R, Rivoltini L, Farina C, Nicolini G, Wolfel T, et al: Cytotoxic T-lymphocyte clones from different patients display limited T-cell-receptor variable-region gene usage in HLA-A2-restricted recognition of the melanoma antigen Melan-A/MART-1. Proc Natl Acad Sci USA 92: 5674-5678, 1995.

18. Salvi S, Segalla F, Rao S, Arienti F, Sartori M, Bratina G, Caronni E, Anichini A, Clemente C, Parmiani G, et al: Overexpression of the $\mathrm{T}$-cell receptor beta-chain variable region TCRBV14 in HLA-A2-matched primary human melanomas. Cancer Res 55: 3374-3379, 1995.

19. Ishida H, Nagai T, Sato S, Honda M, Uotani T, Samejima K, Hanaoka T, Akahori T, Takai Y and Seki H: Concomitant sentinel lymph node biopsy leading to abbreviated systematic lymphadenectomy in a patient with primary malignant melanoma of the vagina. Springerplus 4: 102, 2015.

20. Tanaka N, Mimura M, Kimijima Y and Amagasa T: Clinical investigation of amelanotic malignant melanoma in the oral region . J Oral Maxillofac Surg 62: 933-937, 2004.

21. Ariel IM: Amelanotic melanomas: An analysis of 77 patients. Curr Surg 38: 151-155, 1981.

22. Fujita S, Takahashi $\mathrm{H}$, Tsuda $\mathrm{N}$ and Okabe $\mathrm{H}$ : Immunohistochemical localization of S-100 protein and its subunits in melanotic lesion in the oral mucosa and skin. J Oral Pathol Med 20: 429-432, 1991.

23. Naoki $\mathrm{O}$ and Kawada: The stage of melanogenesis in amelanotic melanoma. In: Melanoma in the Clinic - Diagnosis, Management and Complications of Malignancy. Murph M (ed.) InTech, pp277-283, 2011.

24. Naoki O, Masuki Y, Shigeru K and Akira K: Amelanotic vulvar melanoma with intratumor histological heterogeneity. J Dermatol 37: 537-541, 2010. 